\title{
LOCAL PROJECTION STABILIZATION FOR THE OSEEN PROBLEM AND ITS INTERPRETATION AS A VARIATIONAL MULTISCALE METHOD*
}

\author{
M. BRAACK ${ }^{\dagger}$ AND E. BURMAN ${ }^{\ddagger}$
}

\begin{abstract}
We propose to apply the recently introduced local projection stabilization to the numerical computation of the Oseen equation at high Reynolds number. The discretization is done by nested finite element spaces. Using a priori error estimation techniques, we prove the convergence of the method. The a priori estimates are independent of the local Peclet number and give a sufficient condition for the size of the stabilization parameters in order to ensure optimality of the approximation when the exact solution is smooth. Moreover, we show how this method may be cast in the framework of variational multiscale methods. We indicate what modeling assumptions must be made to use the method for large eddy simulations.
\end{abstract}

Key words. stabilized finite elements, Galerkin methods, multiscale

AMS subject classifications. 35Q30, 65N12, 65N30, 76D05, 76M10, 76F65

DOI. $10.1137 / 050631227$

1. Introduction. In this paper, we advocate the use of the two-level stabilization scheme (see Becker and Braack [3]) for the computation of solutions of the Navier-Stokes equations at high Reynolds number. This is one in a group of more recently developed stabilized methods, such as, for instance, Guermond [14], Becker and Braack [2], Burman and Hansbo [5, 6], and Rebollo and Delgado [18].

A main advantage of this approach is that it shares similar conservation properties with a standard Galerkin finite element method. Moreover, one does not need to resort to space-time finite elements for time stepping in order to stay consistent but can apply any higher-order finite difference scheme for the discretization in time. We prove optimal order a priori error estimates for the method. The method remains stable independent of the local Reynolds number. It should be noted that the method is not residual based in the way the streamlined upwind Petrov-Galerkin (SUPG) method is, but is flexible with respect to the subgrid model, thus leaving open the possibility of subgrid models that are more complex than the linear one considered here. Of course, the possibility of using Galerkin least-squares (GLS) or residual-free bubbles as a subgrid model remains and will be discussed.

An attractive feature of this method is that it can be cast in the framework of the variational multiscale (VMS) method of [15] as we shall show. The stabilization is acting only on the smallest resolved scales of the flow. Hence, contrary to the method of [15], our large scales do not need any additional stabilization. In fact, the fine scale

*Received by the editors May 11, 2005; accepted for publication (in revised form) June 22, 2005; published electronically DATE. This work was initiated while the first author was a guest of the Bernoulli Center program on Mathematical Modelling for Cardiovascular Flows; it was completed while the second author was invited by the "Sonderforschungsbereich 359: Reactive Flow, Diffusion and Transport" at the University of Heidelberg. The support of both institutions is gratefully acknowledged.

http://www.siam.org/journals/sinum/x-x/63122.html

${ }^{\dagger}$ Institute of Applied Mathematics, University of Heidelberg, INF 294, 69120 Heidelberg, Germany (malte.braack@iwr.uni-heidelberg.de).

¥École Polytechnique Fédérale de Lausanne, Institute of Analysis Modelling and Scientific Computing, CH-1015 Lausanne, Switzerland (erik.burman@epfl.ch). 
fluctuations allow for both the satisfaction of the inf-sup condition and stabilization of the convective terms.

We begin in section 2 with the variational formulation of the Oseen equations and their discretization by finite elements in space. The local projection stabilization for the Oseen system is formulated in section 3. Afterwards, an a priori error analysis is presented in section 4 . There we consider the case of smooth solutions (velocities and pressure are both in the Sobolev space $H^{2}(\Omega)$ ) and discuss the behavior of the method for less regular solutions. We present some variants of the stabilization operator and discuss the relation to more standard stabilization techniques in section 5 . An interpretation of the stabilization in terms of a VMS method is given in section 6 . In a numerical test case, discussed in section 7 , we investigate the convergence order for a given exact Navier-Stokes solution and compare the kinetic energy of a nonstationary driven cavity flow with the numerical dissipation. We finish with a short conclusion in section 8. In forthcoming work we will give numerical evidence of the performance of the numerical scheme for turbulent flow in three space dimensions.

2. Variational formulation of the Oseen system. Let $\Omega \subset \mathbb{R}^{d}, d \in\{2,3\}$, be a polygonal domain with boundary $\partial \Omega$. The velocities will be denoted by $v=v(x, t)$ and the pressure by $p=p(x, t)$. The gradient in space is denoted by $\nabla$, and the divergence with respect to space is denoted by div. The Navier-Stokes equations read

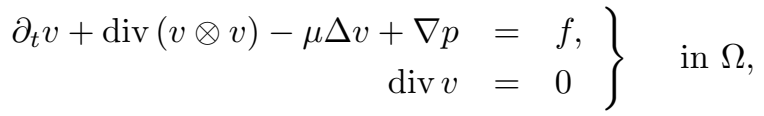

subject to some initial condition $v(\cdot, 0)=v_{0}$ and suitable boundary conditions for $v$.

In the analysis, we will consider the Oseen system as an important linearization of (2.1). For ease of presentation, we suppose homogeneous Dirichlet boundary conditions:

$$
\left.\begin{array}{rlll}
\sigma v+\operatorname{div}(\beta \otimes v)-\mu \Delta v+\nabla p & =f & \text { in } \Omega, \\
\operatorname{div} v & =0 & \text { in } \Omega, \\
v & =0 & \text { on } \partial \Omega,
\end{array}\right\}
$$

with some given solenoidal vector field $\beta$ and $\sigma>0$.

For the variational formulation we use the notation $(\cdot, \cdot)$ for the $L^{2}$-scalar product over $\Omega$. The velocity is sought in the Sobolev space $V:=\left[H_{0}^{1}(\Omega)\right]^{d}$, and the pressure is sought in the space of square-integrable functions with zero mean, $Q:=L_{0}^{2}(\Omega)$. The product space for the vector $u=\{v, p\}$ is denoted by $X:=V \times Q$.

We introduce the bilinear form $A(u, \varphi)$ defined by

$$
A(u, \varphi):=(\sigma v, \psi)-(\beta \otimes v, \nabla \psi)-(p, \operatorname{div} \psi)+(\operatorname{div} v, \xi)+(\mu \nabla v, \nabla \psi)
$$

and consider $f$ as an element of $X^{\prime}$ defined by $\langle f, \varphi\rangle:=(f, \psi)$ for a test function $\varphi=\{\psi, \xi\} \in X$. The variational formulation of the Oseen problem (2.2) reads

$$
u \in X: \quad A(u, \varphi)=\langle f, \varphi\rangle \quad \forall \varphi \in X
$$

In order to solve this problem numerically we choose in the following section a finite dimensional subspace, $X_{h} \subset X$.

2.1. Discrete Galerkin formulation. We consider shape regular meshes $\mathcal{T}_{h}=$ $\{K\}$ of hexaedral elements $K$ with the minimum mesh size $h=\min \left\{h_{K}: K \in \mathcal{T}_{h}\right\}$ 
(quadrilateral elements for the academical case $d=2$ ). The finite element spaces $Q_{h}^{r}$ result from isoparametric transformations of polynomials on a reference cell $\hat{K}$ :

$$
Q_{h}^{r}(\Omega, \mathbb{R}):=\left\{\varphi \in C(\Omega, \mathbb{R}):\left.\varphi\right|_{K}=\hat{\varphi} \circ T_{K}^{-1}\right\},
$$

where $\hat{\varphi}$ denotes an arbitrary polynome of maximal degree $r$ on the reference cell $\hat{K}$, and $T_{K}: \hat{K} \rightarrow K$ denotes a polynomial transformation of the same type and degree $r$. We will treat (bi-/tri-)linear elements $(r=1)$ and (bi-/tri-)quadratic elements $(r=2)$ simultaneously in the analysis. These finite element spaces will simply be called $Q_{1}$ in the case of $r=1$ and $Q_{2}$ elements in the case $r=2$. The discrete pressure space $Q_{h}$ is the subspace of $Q_{h}^{r}$ with zero mean, and the velocity space $V_{h}$ is the subspace with vanishing trace:

$$
Q_{h}:=Q \cap Q_{h}^{r}(\Omega, \mathbb{R}), \quad V_{h}:=V \cap\left[Q_{h}^{r}\left(\Omega, \mathbb{R}^{d}\right)\right]^{d} .
$$

The product space is denoted by $X_{h}$ :

$$
X_{h}:=V_{h} \times Q_{h} .
$$

In the Galerkin formulation of (2.3) for the space $X_{h}$, a discrete solution $u_{h} \in X_{h}$ is sought such that

$$
A\left(u_{h}, \varphi\right)=\langle f, \varphi\rangle \quad \forall \varphi \in X_{h} .
$$

This formulation is not stable due to the following reasons: (i) violation of the discrete inf-sup (or Babuska-Brezzi) condition for velocity and pressure approximation and (ii) dominating advection (and reaction). Therefore, in the following we present and analyze a stabilization technique based on local projection.

3. Definition of the local projection stabilization. The two-level finite element formulation is as follows: find $u_{h} \in X_{h}$ such that

$$
A\left(u_{h}, \varphi\right)+S_{h}\left(u_{h}, \varphi\right)=\langle f, \varphi\rangle \quad \forall \varphi \in X_{h} .
$$

In order to specify the stabilization term $S_{h}(\cdot, \cdot)$, we have to introduce further notations. The discontinuous analogon of $Q_{h}^{r}$ is denoted by $Q_{h, d i s c}^{r}$ :

$$
Q_{h, d i s c}^{r}(\Omega, \mathbb{R}):=\left\{\varphi \in L^{2}(\Omega, \mathbb{R}):\left.\varphi\right|_{K}=\hat{\varphi} \circ T_{K}^{-1}\right\} .
$$

Furthermore, let $\mathcal{T}_{2 h}$ be the coarser mesh obtained by a "global coarsening" of $\mathcal{T}_{h}$. Obviously, the finer mesh $\mathcal{T}_{h}$ contains $2^{d}$ times more elements than $\mathcal{T}_{2 h}$. The corresponding finite element spaces are denoted by $Q_{2 h} \subset Q_{h}$ and $V_{2 h} \subset V_{h}$. Let $D_{h}^{v}$ and $D_{h}^{p}$ be the following space for pressure and velocities, respectively, of functions allowing discontinuities across elements of $\mathcal{T}_{2 h}$ :

$$
\begin{aligned}
D_{h}^{v} & :=\left[Q_{2 h, d i s c}^{r-1}(\Omega, \mathbb{R})\right]^{d}, \\
D_{h}^{p} & :=Q_{2 h, \text { disc }}^{r-1}(\Omega, \mathbb{R}) .
\end{aligned}
$$

In the case $r=1$, these spaces contain patchwise $\left(K \in V_{2 h}\right)$ constants; for $r=2$, they contain patchwise linear elements. We will make use of the $L^{2}$-projection operator

$$
\bar{\pi}_{h}: L^{2}(\Omega) \rightarrow D_{h}^{p},
$$


characterized by the property

$$
\left(v-\bar{\pi}_{h} v, \phi\right)=0 \quad \forall \phi \in D_{h}^{p} .
$$

The operator giving the space fluctuations is denoted by

$$
\bar{\varkappa}_{h}:=i-\bar{\pi}_{h},
$$

with the identity mapping $i$. We use the same notation $\bar{\pi}_{h}, \bar{\varkappa}_{h}$ for the mappings on vector-valued functions, for instance, $\bar{\pi}_{h}: L^{2}(\Omega)^{d} \rightarrow D_{h}^{v}$.

The subgrid model is given by

$$
S_{h}(u, \varphi):=\left(\delta \bar{\varkappa}_{h} \nabla v, \bar{\varkappa}_{h} \nabla \psi\right)+\left(\alpha \bar{\varkappa}_{h} \nabla p, \bar{\varkappa}_{h} \nabla \xi\right) .
$$

The parameters $\alpha$ and $\delta$ are taken patchwise constant and depend on the local mesh size. The optimal choice of these stabilization parameters will be a result of the following analysis.

We like to end this section with a brief discussion on the numerical costs of the scheme compared to more standard stabilized schemes. Compared to the standard Galerkin formulation, the subgrid models (3.2) lead to a larger stencil in the stiffness matrix due to the projection $\bar{\varkappa}_{h}$. However, no couplings between pressure and velocities are introduced. Furthermore, a cheaper preconditioner may be used with a smaller stencil as proposed and analyzed for the Stokes system in [2].

4. A priori error analysis. To tune the stabilization parameters $\alpha$ and $\delta$ we use a priori error estimation. Assuming sufficient regularity of the underlying solution, the parameters are chosen in such a way that the method has optimal convergence properties independent of the viscosity. We will prove under the assumption of sufficiently regular pressure and velocity $v \in\left[H_{0}^{2}(\Omega)\right]^{3}, p \in H^{2}(\Omega) \cap L_{0}^{2}(\Omega)$, that a certain scaling of $\alpha$ and $\delta$ gives optimal convergence of the velocities independent of the Reynolds number. A similar result is then proved for the $L^{2}$-norm of the pressure. We only treat the interesting case of high Reynolds number, hence assuming that $\mu \leq|\beta| h$. First we prove an estimate for a mesh-dependent norm including the $H^{1}$-norm of the velocities and a "subgrid model" error:

$$
\|u\|=\|\{v, p\}\|:=\left(\left\|\sigma^{1 / 2} v\right\|^{2}+\left\|\mu^{1 / 2} \nabla v\right\|^{2}+S_{h}(u, u)\right)^{1 / 2},
$$

where $\|\cdot\|$ stands for the $L^{2}$-norm in $\Omega$. We then use this estimate to recover control of the pressure and show that the $L^{2}$-norm error of the pressure is bounded by the mesh-dependent norm of the error of the state vector $u_{h}$.

4.1. Properties of the subgrid model. By the following coercivity result we deduce existence and uniqueness of the discrete velocities.

LEMma 4.1. We have the coercivity property

$$
\|u\|^{2}=A(u, u)+S_{h}(u, u) \quad \forall u \in X .
$$

Proof. The proof follows immediately by integration by parts.

We have the following approximate Galerkin orthogonality.

Lemma 4.2. Let $u \in X$ be the solution of the weak formulation of (2.2) and let $u_{h} \in X_{h}$ be the solution of its discrete version (3.1). Then it holds that

$$
A\left(u-u_{h}, \varphi\right)=S_{h}\left(u_{h}, \varphi\right), \quad \varphi \in X_{h} .
$$




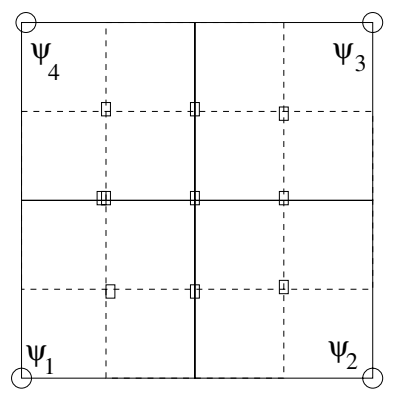

FIG. 4.1. A patch $K$ of four $Q_{2}$ cells in two dimensions with the linears $\psi_{i}$ used in the proof of Lemmas 4.4 and 4.5 . (2.2).

Proof. The proof is obtained by subtracting (3.1) from the weak formulation of

Since the method is not strongly consistent in the sense that we do not have full Galerkin orthogonality, we must analyze the asymptotic behavior of the subgrid model, i.e., the dependence with respect to the mesh size $h$. We first prove a result for a modified Clément interpolation operator introduced in [2], here with a generalization to $P_{2}$ elements, and use this approximation result to show the asymptotic behavior of the stabilization term.

In the next lemma, we consider for a cell $K \in \mathcal{T}_{2 h}$, the space of functions in $Q_{h}$ with support in $K$. This space will be denoted by $Q_{h}(K)$ and has the dimension $(2 r-1)^{d}$. Analogously, the subspace of $D_{h}^{p}$ consisting of functions with support in $K$ will be denoted by $D_{h}^{p}(K)$. This space has the dimension $r^{d}$. For $r=1$, this space consists only of the patchwise constants. For $r=2$, a possible basis of these subspaces is indicated in Figure 4.1.

LEMma 4.3. The local $L^{2}$-orthogonal projection $\pi_{K}: D_{h}^{p}(K) \rightarrow Q_{h}(K)$, characterized for $\psi \in D_{h}^{p}(K)$ by the property

$$
\left(\pi_{K} \psi, \phi\right)=(\psi, \phi) \quad \forall \phi \in Q_{h}(K)
$$

is injective.

Proof. We assume $\pi_{K} \psi=0$ for $\psi \in D_{h}^{p}(K)$. Then $(\psi, \phi)=0$ for all $\phi \in Q_{h}(K)$ due to the orthogonality property. In the case $r=1, D_{h}^{p}(K)$ consists of constant functions, so that either $\psi=0$ or $\int_{K} \phi=0$ for all $\phi \in Q_{h}(K)$. Since the latter is not valid (for instance, taking the Lagrange nodal basis function associated to the interior node of $K$ ), it follows $\psi=0$. For $r=2$, we take as $\phi_{i} \in Q_{h}(K), i=1, \ldots, 2^{d}$, the Lagrange nodal functions associated to the center nodes of the child cells $K_{i}$. Since these $\phi_{i}$ have a sign, $\psi$ must have zeros in the interior of all child cells $K_{i}$, $i=1, \ldots, 2^{d}$. For a $d$-linear $\psi$ this is possible only if $\psi=0$.

Lemma 4.4. Let $\left\{\psi_{1}, \ldots, \psi_{r^{d}}\right\}$ be an arbitrary basis of $D_{h}^{p}(K)$. Then the matrix $M=\left(m_{i j}\right), i, j \in\left\{1, \ldots, r^{d}\right\}$, with entries

$$
m_{i j}=\left(\psi_{i}, \pi_{K} \psi_{j}\right)_{K},
$$

is symmetric and positive definite.

Proof. Since $\pi_{K} \psi_{j} \in Q_{h}(K)$, it follows due to the orthogonality property of the $L^{2}$-projection that

$$
m_{i j}=\left(\psi_{i}, \pi_{K} \psi_{j}\right)=\left(\pi_{K} \psi_{i}, \pi_{K} \psi_{j}\right) .
$$


It follows that $M$ is symmetric. Furthermore, for $\alpha \in \mathbb{R}^{r^{d}}$ and $\psi_{\alpha}=\sum \alpha_{i} \psi_{i}$ it holds that

$$
\begin{aligned}
\alpha^{T} M \alpha & =\left(\psi_{\alpha}, \pi_{K} \psi_{\alpha}\right)_{K} \\
& =\left(\pi_{K} \psi_{\alpha}, \pi_{K} \psi_{\alpha}\right)_{K} \\
& =\left\|\pi_{K} \psi_{\alpha}\right\|_{K}^{2} .
\end{aligned}
$$

Since $\pi_{K}$ is injective, $M$ is positive definite.

Remark. The analogous results are valid for vector-valued projections $\pi_{K}$ : $D_{h}^{v}(K) \rightarrow V_{h}(K)$, where $D_{h}^{v}(K) \subset D_{h}^{v}$ and $V_{h}(K) \subset V_{h}$ are defined analogously as $D_{h}^{p}(K)$ and $Q_{h}(K)$, respectively.

In the following, the norm in $H^{s}(\Omega)$ will be denoted by $\|\cdot\|_{s}$. The corresponding norms in subsets $K \subset \Omega$ will be denoted by $\|\cdot\|_{s, K}$. Furthermore, we use the notation $\lesssim$ to indicate that there may arise mesh-independent constants in the estimates.

LEMma 4.5. There is an interpolation operator

$$
j_{h}: V \rightarrow V_{h}
$$

with the orthogonality property

$$
\left(v-j_{h} v, \psi\right)=0 \quad \forall \psi \in D_{h}^{v}, \forall v \in V
$$

that has optimal approximation properties in the $L^{2}$-norm and $H^{1}$-seminorm

$$
\begin{aligned}
\left\|v-j_{h} v\right\| & \lesssim h^{l}\|v\|_{l} \quad \forall v \in\left[H^{l}(\Omega)\right]^{d}, 0 \leq l \leq r+1, \\
\left\|\nabla\left(v-j_{h} v\right)\right\| & \lesssim h^{l-1}\|v\|_{l} \quad \forall v \in\left[H^{l}(\Omega)\right]^{d}, 0 \leq l \leq r+1,
\end{aligned}
$$

with $r \in\{1,2\}$, and is $H^{1}$-stable:

$$
\left\|j_{h} v\right\|_{1} \lesssim\|v\|_{1} \quad \forall v \in\left[H^{1}(\Omega)\right]^{d} .
$$

Proof. The construction uses the Scott and Zhang variant of the Clément interpolation operator $j_{h}^{C l}: V \rightarrow V_{h}$ (see [19] and Clément [8]), which already fulfills the approximation properties (4.3) and (4.4), maintains homogeneous Dirichlet values, and has the stability property (4.5). In order to ensure (4.2), we define $j_{h}$ in the form

$$
j_{h}=j_{h}^{C l}+m_{h}
$$

with a local projection $m_{h}: V \rightarrow \tilde{V}_{h}$ onto the subspace

$$
\tilde{V}_{h}:=\bigoplus_{K \in \mathcal{T}_{2 h}} V_{h}(K) \subset V_{h} .
$$

In order to fulfill (4.2) this mapping must satisfy

$$
\left(m_{h} v, \psi\right)=\left(v-j_{h}^{C l} v, \psi\right), \quad \forall \psi \in D_{h}^{v}(K), \forall K \in \mathcal{T}_{2 h} .
$$

If we take a basis $\psi_{K, i}$ of $D_{h}^{v}(K), m_{h} v$ can be expressed on each patch $K \in \mathcal{T}_{2 h}$ as a linear combination of the $\pi_{K} \psi_{K, i}$. Hence property (4.6) is equivalent to solving for each $K$ the linear system $M \alpha=\beta$, with the regular matrix $M$ of Lemma 4.4 and the right-hand side $\beta$ with coefficients

$$
\beta_{i}=\left(v-j_{h}^{C l} v, \psi_{K, i}\right)_{K} .
$$


Hence, $j_{h}$ is well defined. In order to prove the approximation property in the $L^{2}$ norm (4.3) we will show that

$$
\left\|m_{h} v\right\|_{K} \lesssim\left\|v-j_{h}^{C l} v\right\|_{K}
$$

because then we follow:

$$
\begin{aligned}
\left\|v-j_{h} v\right\| & =\left\|v-j_{h}^{C l} v+m_{h} v\right\| \\
& \leq\left\|v-j_{h}^{C l} v\right\|+\left\|m_{h} v\right\| \\
& \lesssim\left\|v-j_{h}^{C l} v\right\| .
\end{aligned}
$$

Let us verify (4.7): The ( $v$-dependent) solution vector $\alpha$ contains the coefficients of $m_{h} v$ in the basis $\pi_{K} \psi_{K, i}$. Similarly, the projection of $m_{h} v$ onto the vectors $\psi_{K, i}$ can be expressed as a linear combination of the $\psi_{K, i}$ with coefficients $\bar{\alpha}_{K, i}$ :

$$
\bar{\pi}_{h} m_{h} v=\sum_{i=1}^{r^{d}} \bar{\alpha}_{K, i} \psi_{K, i} .
$$

Now, the equality

$$
\left(\bar{\pi}_{h} m_{h} v, \psi_{K, i}\right)_{K}=\left(m_{h} v, \psi_{K, i}\right)_{K}, \quad i=1, \ldots, r^{d},
$$

can be written with the help of the matrix $N=\left(n_{i j}\right)$ with coefficients $n_{i j}=\left(\psi_{K, i}, \psi_{K, j}\right)$ :

$$
N \bar{\alpha}=M \alpha .
$$

The local mass matrix $N$ is symmetric and positive definite as well. It then follows that

$$
\begin{aligned}
\lambda_{\min }(M)\left\|m_{h} v\right\|_{K}^{2} & \leq(M \alpha)^{T} \cdot M \alpha \\
& =(N \bar{\alpha})^{T} N \bar{\alpha} \\
& \leq \lambda_{\max }(N)\left\|\bar{\pi}_{h} m_{h} v\right\|_{K}^{2} .
\end{aligned}
$$

We conclude that there holds on each patch $K \in \mathcal{T}_{2 h}$

$$
\left\|m_{h} v\right\|_{K}^{2} \lesssim\left\|\bar{\pi}_{h} m_{h} v\right\|_{K}^{2}=\left(m_{h} v, \bar{\pi}_{h}\left(m_{h} v\right)\right)_{K},
$$

where the constant is independent of the mesh size. We estimate on each patch $K \in \mathcal{T}_{2 h}$ due to (4.9):

$$
\begin{aligned}
\left\|m_{h} v\right\|_{K}^{2} & \lesssim \int_{K}\left(v-j_{h}^{C l} v\right) \bar{\pi}_{h} m_{h} v d x \\
& \leq\left\|v-j_{h}^{C l} v\right\|_{K}\left\|\bar{\pi}_{h} m_{h} v\right\|_{K} \\
& \lesssim\left\|v-j_{h}^{C l} v\right\|_{K}\left\|m_{h} v\right\|_{K},
\end{aligned}
$$

where we used the $L^{2}$-stability of $\bar{\pi}_{h}$ in the last inequality. It follows (4.7).

For proving (4.4) we proceed in a similar fashion by applying an inverse estimate:

$$
\begin{aligned}
\left\|\nabla m_{h} v\right\|^{2} & \lesssim \sum_{K \in \mathcal{T}_{h}} h_{K}^{-2}\left\|m_{h} v\right\|_{K}^{2} \\
& =\sum_{K \in \mathcal{T}_{h}} h_{K}^{-2}\left\|v-j_{h}^{C l} v\right\|_{K}^{2} \\
& \lesssim \sum_{K \in \mathcal{T}_{h}} h_{K}^{2(l-1)}\|v\|_{l, \widetilde{K}}^{2} \\
& \lesssim h^{2(l-1)}\|v\|_{l}^{2} .
\end{aligned}
$$


The stability (4.5) follows also due to this last estimate, the stability of $j_{h}^{C L}$, and (4.7).

Remark. The interpolation operator $j_{h}^{C l}$ maintains homogeneous Dirichlet conditions on (parts of) $\partial \Omega$. For polynomial Dirichlet condition, the interpolation introduced by Melenk and Wohlmuth [16] can be used. The interpolation operator $j_{h}$ acts on the velocity space, but the result holds true of course for the scalar space $L^{2}(\Omega)$. We will therefore use the notation $j_{h}$ also for the interpolation operator acting on the state variable $u=\{v, p\}$.

In the following analysis, we make use of the interpolation and stability properties of $\bar{\varkappa}_{h}$.

LEMMA 4.6. The fluctuation operator $\bar{\varkappa}_{h}$ has the following interpolation and stability properties:

$$
\begin{aligned}
\left\|\bar{\varkappa}_{h} \nabla v\right\| & \lesssim h^{r}\|v\|_{r+1} \quad \forall v \in H^{r+1}(\Omega), \\
\left\|\bar{\varkappa}_{h} v\right\| & \lesssim\|v\| \quad \forall v \in L^{2}(\Omega) .
\end{aligned}
$$

Proof. The interpolation property (4.10) is an immediate consequence of the patch-wise interpolation of $\bar{\pi}_{h}$ for the $H^{1}$ function $w:=\nabla v$ :

$$
\left\|\bar{\varkappa}_{h} \nabla v\right\|_{K}=\left\|w-\bar{\pi}_{h} w\right\|_{K} \lesssim h_{K}^{r}\|w\|_{r, K} \leq h_{K}^{r}\|v\|_{r+1, K} \quad \forall K \in \mathcal{T}_{2 h}
$$

Stability of $\bar{\varkappa}_{h}$ is due to the $L^{2}$-stability of $\bar{\pi}_{h}$ :

$$
\left\|\bar{\varkappa}_{h} v\right\| \leq\|v\|+\left\|\bar{\pi}_{h} v\right\| \lesssim\|v\|
$$

LEMmA 4.7. For the interpolation operator $j_{h}$ of Lemma 4.5 we have for all $u \in X \cap\left[H^{r+1}(\Omega)\right]^{d+1}$

$$
S_{h}\left(j_{h} u, j_{h} u\right)^{1 / 2} \lesssim\left(\delta^{1 / 2}+\alpha^{1 / 2}\right) h^{r}\left(\|v\|_{r+1}+\|p\|_{r+1}\right) .
$$

Proof. We start with adding and subtracting $u$ :

$$
\begin{aligned}
S_{h}\left(j_{h} u, j_{h} u\right) & =S_{h}\left(u+j_{h} u-u, u+j_{h} u-u\right) \\
& \leq S_{h}(u, u)+S_{h}\left(j_{h} u-u, j_{h} u-u\right)+2 S_{h}\left(j_{h} u-u, u\right) \\
& \leq 2\left(S_{h}(u, u)+S_{h}\left(j_{h} u-u, j_{h} u-u\right)\right) .
\end{aligned}
$$

For the first term the result follows immediately by the interpolation property (4.10):

$$
\begin{aligned}
S_{h}(u, u) & \leq \delta\left\|\bar{\varkappa}_{h} \nabla v\right\|^{2}+\alpha\left\|\bar{\varkappa}_{h} \nabla p\right\|^{2} \\
& \lesssim \delta h^{2 r}\|v\|_{r+1}^{2}+\alpha h^{2 r}\|p\|_{r+1}^{2}
\end{aligned}
$$

For the second term $S_{h}\left(j_{h} u-u, j_{h} u-u\right)$ we have

$$
\begin{aligned}
\delta\left\|\bar{\varkappa}_{h} \nabla\left(j_{h} v-v\right)\right\|^{2} & \lesssim \delta\left\|\nabla\left(j_{h} v-v\right)\right\|^{2} \\
& \lesssim \delta h^{2 r}\|v\|_{r+1}^{2}
\end{aligned}
$$

using the $L^{2}$-stability (4.11) of the local projector $\bar{\varkappa}_{h}$ and the interpolation property (4.4) of $j_{h}$. For the pressure contribution of course the same holds.

4.2. A priori estimate for smooth velocities and pressure. In this subsection, we prove the following a priori estimate for the discrete solution of (3.1). 
THEOREM 4.8. If the solution $u=\{v, p\}$ of (2.2) satisfies $u \in\left[H^{r+1}(\Omega)\right]^{d+1}$, then we have the a priori estimate

$$
\left\|u-u_{h}\right\| \lesssim a h^{r+\frac{1}{2}}\left(\|v\|_{r+1}+\|p\|_{r+1}\right)
$$

with

$$
a=h^{-1 / 2}\left(\mu^{1 / 2}+\delta^{1 / 2}+\alpha^{1 / 2}\right)+h^{1 / 2}\left(\sigma^{1 / 2}+\delta^{-1 / 2}+\alpha^{-1 / 2}\right) .
$$

Before proceeding with the proof of this theorem, let us briefly comment on its interpretation. An immediate consequence of the inequality (4.12) is that for convection dominated flow, $\delta \sim h$ and $\alpha \sim h$ is the optimal choice of the parameters yielding an $h$-independent constant $a$ and the (optimal) convergence order of $h^{r+1 / 2}$. The positive powers of $\delta$ and $\alpha$ in (4.13) represent the dissipative character of the stabilization terms. It follows that too much dissipation will have a negative effect on the precision. The presence of $\delta^{-1 / 2}$ and $\alpha^{-1 / 2}$ in (4.13) is due to the stabilizing effect of the subgrid model: The dissipation of the small-scale energy into the unresolved scales avoids artificial energy concentrations on the small scales due to the conservation properties of the Galerkin method. As expected, precision deteriorates for small values of $\delta$ and $\alpha$ due to spurious oscillations.

Proof. In the standard fashion we decompose the error in $u-u_{h}=\eta+\xi$ into an interpolation part $\eta=u-j_{h} u$ and a projection part $\xi=j_{h} u-u_{h}$. Clearly, $\|\eta\| \leq C a h^{r+1 / 2}$ using the interpolation Lemma 4.5 and the asymptotic bound for the stabilization term of Lemma 4.7. Consider now the discrete error $\xi$. By coercivity (Lemma 4.1) and the Galerkin orthogonality property (Lemma 4.2) we have

$$
\begin{aligned}
\|\xi\|^{2} & =A(\xi, \xi)+S_{h}(\xi, \xi) \\
& =A(\eta, \xi)+S_{h}\left(j_{h} u, \xi\right) .
\end{aligned}
$$

The second term on the right-hand side is bounded by applying the Cauchy-Schwarz inequality followed by Lemma 4.7:

$$
\begin{aligned}
S_{h}\left(j_{h} u, \xi\right) & \leq S_{h}\left(j_{h} u, j_{h} u\right)^{1 / 2} S_{h}(\xi, \xi)^{1 / 2} \\
& \lesssim\left(\mu^{1 / 2}+\alpha^{1 / 2}\right) h^{r}\left(\|v\|_{r+1}+\|p\|_{r+1}\right)\|\xi\| .
\end{aligned}
$$

For the first term on the right-hand side we use the Cauchy-Schwarz inequality and integration by parts, writing $\xi^{p}$ and $\xi^{v}$ for the discrete pressure and the velocity error, respectively:

$$
\text { (4.14) } A(\eta, \xi) \leq\|\eta\|\|\xi\|-\left(p-j_{h} p, \operatorname{div} \xi^{v}\right)-\left(v-j_{h} v, \nabla \xi^{p}\right)-\left(\beta \otimes\left(v-j_{h} v\right), \nabla \xi^{v}\right) .
$$

We now use the orthogonality property of the quasi-interpolation operator to obtain upper bounds:

$$
\begin{aligned}
\left|\left(p-j_{h} p, \operatorname{div} \xi^{v}\right)\right| & =\left|\left(p-j_{h} p, \operatorname{div} \xi^{v}-\bar{\pi} \operatorname{div} \xi^{v}\right)\right| \\
\leq & \left\|\delta^{-1 / 2}\left(p-j_{h} p\right)\right\|\left\|\delta^{1 / 2}\left(\operatorname{div} \xi^{v}-\bar{\pi} \operatorname{div} \xi^{v}\right)\right\| \\
\leq & \left\|\delta^{-1 / 2}\left(p-j_{h} p\right)\right\| S_{h}(\xi, \xi)^{1 / 2}, \\
\left|\left(v-j_{h} v, \nabla \xi^{p}\right)\right| & =\left(v-j_{h} v, \nabla \xi^{p}-\bar{\pi} \nabla \xi^{p}\right) \\
& \leq\left\|\alpha^{-1 / 2}\left(v-j_{h} v\right)\right\|\left\|\alpha^{1 / 2}\left(\nabla \xi^{p}-\bar{\pi} \nabla \xi^{p}\right)\right\| \\
& \leq\left\|\alpha^{-1 / 2}\left(v-j_{h} v\right)\right\| S_{h}(\xi, \xi)^{1 / 2}, \\
\left|\left(\beta \otimes\left(v-j_{h} v\right), \nabla \xi^{v}\right)\right| & =\left|\left(v-j_{h} v,(\beta \cdot \nabla) \xi^{v}-\bar{\pi}(\beta \cdot \nabla) \xi^{v}\right)\right| \\
& \leq\left\|\delta^{-1 / 2}\left(v-j_{h} v\right)\right\| S_{h}(\xi, \xi)^{1 / 2} .
\end{aligned}
$$


In summary, we get

$$
\begin{aligned}
A(\eta, \xi) & \leq\|\eta\|\|\xi\|+\left(\left\|\delta^{-1 / 2}\left(p-j_{h} p\right)\right\|+\left\|\left(\alpha^{-1 / 2}+\delta^{-1 / 2}\right)\left(v-j_{h} v\right)\right\|\right) S_{h}(\xi, \xi)^{1 / 2} \\
& \leq\left(\|\eta\|+\left\|\delta^{-1 / 2}\left(p-j_{h} p\right)\right\|+\left\|\left(\alpha^{-1 / 2}+\delta^{-1 / 2}\right)\left(v-j_{h} v\right)\right\|\right)\|\xi\| .
\end{aligned}
$$

The assertion follows using interpolation properties (4.3) and (4.4) of the quasi interpolant $j_{h}$.

We proceed and prove that the pressure also has optimal convergence properties in the $L^{2}$-norm.

LEMMA 4.9. Let $u=\{v, p\}$ be the solution of (2.2) and let $u_{h}=\left\{v_{h}, p_{h}\right\}$ be the solution of (3.1). Then there holds

$$
\left\|p-p_{h}\right\| \lesssim a\left\|u-u_{h}\right\|,
$$

where $a=\sigma^{1 / 2}+|\beta| \sigma^{-1 / 2}+\mu^{1 / 2}+\delta^{1 / 2}+\alpha^{-1 / 2} h$.

Proof. Following [12], by the surjectivity of the divergence operator there exists $v_{p} \in\left[H_{0}^{1}(\Omega)\right]^{d}$ such that $p-p_{h}=\operatorname{div} v_{p}$ and $\left\|v_{p}\right\|_{1, \Omega} \lesssim\left\|p-p_{h}\right\|$. By the $H^{1}$-stability property of the quasi interpolant $j_{h}$ we then have

$$
\left\|j_{h} v_{p}\right\|_{1, \Omega} \lesssim\left\|p-p_{h}\right\| .
$$

Consider now the equality $p-p_{h}=\operatorname{div} v_{p}$. This gives

$$
\left\|p-p_{h}\right\|^{2}=\left(p-p_{h}, \operatorname{div} v_{p}\right) .
$$

We now subtract $j_{h} v_{p}$ from $v_{p}$ in the right-hand side and use the Galerkin orthogonality property in Lemma 4.2 for the test function $\left\{j_{h} v_{p}, 0\right\}$ :

$$
\begin{aligned}
\left\|p-p_{h}\right\|^{2}= & \left(p-p_{h}, \operatorname{div}\left(v_{p}-j_{h} v_{p}\right)\right)-\left(\mu \nabla\left(v-v_{h}\right), \nabla j_{h} v_{p}\right) \\
& +\left(\sigma\left(v-v_{h}\right), j_{h} v_{p}\right)+\left(\beta \otimes\left(v-v_{h}\right), \nabla\left(j_{h} v_{p}\right)\right)-S_{h}\left(u-u_{h},\left\{j_{h} v_{p}, 0\right\}\right) .
\end{aligned}
$$

We estimate the resulting parts separately. For the first term we integrate by parts and use the orthogonality property (4.2) of the quasi-interpolation operator $j_{h}$ to obtain

$$
\begin{aligned}
\left(p-p_{h}, \operatorname{div}\left(v_{p}-j_{h} v_{p}\right)\right) & =\left(\nabla\left(p-p_{h}\right), v_{p}-j_{h} v_{p}\right) \\
& =\left(\bar{\varkappa}_{h} \nabla\left(p-p_{h}\right), v_{p}-j_{h} v_{p}\right) \\
& \leq S_{h}\left(\left\{0, p-p_{h}\right\},\left\{0, p-p_{h}\right\}\right)^{1 / 2}\left\|\alpha^{-1 / 2}\left(v_{p}-j_{h} v_{p}\right)\right\| \\
& \lesssim \alpha^{-1 / 2} h\left\|u-u_{h}\right\|\left\|v_{p}\right\|_{1} \\
& \lesssim \alpha^{-1 / 2} h\left\|u-u_{h}\right\|\left\|p-p_{h}\right\|,
\end{aligned}
$$

where we used the stability property of $v_{p}$ in the last inequality. Furthermore, we have

$$
\begin{aligned}
& \left(\sigma\left(v-v_{h}\right), j_{h} v_{p}\right)+\left(\beta \otimes\left(v-v_{h}\right), \nabla\left(j_{h} v_{p}\right)\right) \\
= & \left(\sigma\left(v-v_{h}\right), j_{h} v_{p}\right)-\left(v-v_{h},(\beta \cdot \nabla) j_{h} v_{p}\right) \\
\leq & \left(\sigma^{1 / 2}+|\beta| \sigma^{-1 / 2}\right)\left\|u-u_{h}\right\|\left\|j_{h} v_{p}\right\|_{1} .
\end{aligned}
$$

Similarly we obtain, after application of the Cauchy-Schwarz inequality and (4.11),

$$
\begin{aligned}
& \left(\mu \nabla\left(v-v_{h}\right), \nabla j_{h} v_{p}\right)-S_{h}\left(u-u_{h},\left\{j_{h} v_{p}, 0\right\}\right) \\
\leq & \left\|\mu^{1 / 2} \nabla\left(v-v_{h}\right)\right\|\left\|\mu^{1 / 2} \nabla j_{h} v_{p}\right\|+S_{h}\left(u-u_{h}, u-u_{h}\right)^{1 / 2} S_{h}\left(\left\{j_{h} v_{p}, 0\right\},\left\{j_{h} v_{p}, 0\right\}\right)^{1 / 2} \\
\leq & \left\|u-u_{h}\right\|\left(\mu^{1 / 2}+\delta^{1 / 2}\right)\left\|j_{h} v_{p}\right\|_{1} .
\end{aligned}
$$

Collecting terms and using (4.15) gives the assertion. 
Corollary 4.10. For the solution of (3.1) there holds

$$
\left\|p_{h}\right\|^{2} \lesssim\left\|u_{h}\right\|^{2}+\|f\|^{2}=\left(f, v_{h}\right)+\|f\|^{2} .
$$

Hence the pressure is unique.

Proof. Modifying the proof of Lemma 4.9 considering not $p-p_{h}$ but simply $p_{h}$ and introducing the right-hand side instead of using Galerkin orthogonality, we have

$$
\left\|p_{h}\right\| \lesssim a\left\|u_{h}\right\|+\|f\|
$$

and conclude by applying Lemma 4.1.

4.3. Lower regularities. The aim of the smoothness assumptions above is to show that the discretization allows for the quasi-optimal a priori error estimates that are characteristic for stabilized methods. However, for the case of high Reynolds number flows this may seem overly optimistic, and we will therefore discuss what we may prove rigorously in the case where the pressure is only in $H^{1}(\Omega)$ and the velocities are in $\left[H^{2}(\Omega)\right]^{d}$. In order to recover optimality of the estimate, the pressure stabilization has to be reduced to $\alpha \sim h^{2}$. Otherwise, the error would be dominated by the term $\alpha^{1 / 2}\|p\|_{1}$, which would lead to a convergence order of only $h^{1 / 2}$.

Lemma 4.11. In the case of less regular velocities and pressure, $v \in\left[H^{2}(\Omega)\right]^{d}$ and $p \in H^{1}(\Omega)$, and the use of the stabilization

$$
S_{h}(u, \varphi)=\left(\delta \nabla \varkappa_{h} v, \nabla \varkappa_{h} \psi\right)+\left(\alpha \nabla \varkappa_{h} p, \nabla \varkappa_{h} \xi\right)+\left(\bar{\varkappa}_{h} \operatorname{div} v_{h}, \bar{\varkappa}_{h} \operatorname{div} \psi\right),
$$

with $\delta \sim h$ and $\alpha \sim h^{2}$, it holds for $v \in\left[H^{2}(\Omega)\right]^{d}, p \in H^{1}(\Omega)$, and high local Peclet number that

$$
\left\|u-u_{h}\right\| \lesssim h^{3 / 2}\|v\|_{2}+h\|p\|_{1} .
$$

Furthermore, for lower regularity $v \in\left[H^{1+\epsilon}(\Omega)\right]^{d}, p \in L^{2}(\Omega)$ with $\epsilon>0$ and under the assumption

$$
\left\|v-j_{h} v\right\| \lesssim h^{1+\epsilon}\|v\|_{1+\epsilon},
$$

we have at least convergence $\left\|u-u_{h}\right\| \rightarrow 0$ for $h \rightarrow 0$.

Proof. We begin with the case $v \in\left[H^{2}(\Omega)\right]^{d}, p \in H^{1}(\Omega)$. The regularity of the pressure is necessary only for the upper bound of the stabilizing term of Lemma 4.7. The lower regularity gives the modified upper bound

$$
S_{h}\left(j_{h} u, j_{h} u\right)^{1 / 2} \lesssim \delta^{1 / 2} h\|v\|_{2}+\alpha^{1 / 2}\|p\|_{1} .
$$

Due to the decrease of the pressure stabilization to $\alpha \sim h^{2}$ it follows from the proof of Theorem 4.8 that the control of the incompressibility condition has to be increased. This is warranted due to the additional stabilization term

$$
\left\|\bar{\varkappa}_{h} \operatorname{div} v_{h}\right\| \leq S_{h}\left(u_{h}, u_{h}\right) .
$$

Hence, we deduce

$$
\begin{aligned}
\left|\left(p-j_{h} p, \operatorname{div} \xi^{v}\right)\right| & =\left|\left(p-j_{h} p, \operatorname{div} \xi^{v}-\bar{\pi} \operatorname{div} \xi^{v}\right)\right| \\
& \leq\left\|p-j_{h} p\right\| S_{h}\left(u_{h}, u_{h}\right) .
\end{aligned}
$$


For the case $v \in H^{1+\epsilon}(\Omega)$ and $p \in L^{2}(\Omega)$, of course we cannot expect to get any convergence order. On the other hand, the choice $\alpha \sim h^{2}$ and (4.17) allow us to prove convergence by a density argument, provided that the interpolants converge. The Scott-Zhang operator is no longer well defined on the space of $L^{2}$-functions, and we therefore replace it by the $L^{2}$-projection. Assuming quasi-uniform meshes, the same estimate holds. First we check the stabilization operator $S\left(j_{h} u, j_{h} u\right)$. Using the stability of the projection $\bar{\varkappa}_{h}$, the $L^{2}$-projection onto a piecewise constant on element $K, \pi_{0, K}$, and an inverse inequality, we deduce that

$$
\begin{aligned}
S\left(j_{h} u, j_{h} u\right) & \lesssim \sum_{K}\left(\delta_{K}\left\|\nabla j_{h} v\right\|_{K}^{2}+\alpha_{K}\left\|\nabla j_{h} p\right\|_{K}^{2}\right) \\
& \lesssim \sum_{K}\left(h_{K}\|\nabla v\|_{K}^{2}+\left\|j_{h} p-\pi_{0, K} p\right\|_{K}^{2}\right) \\
& \lesssim \sum_{K}\left(h_{K}\|\nabla v\|_{K}^{2}+\left\|p-j_{h} p\right\|_{K}^{2}+\left\|p-\pi_{0, K} p\right\|_{K}^{2}\right) \\
& \rightarrow 0 \quad(\text { for } h \rightarrow 0) .
\end{aligned}
$$

Convergence of the other terms are achieved in a similar fashion assuming (4.16).

In the case of low local Reynolds number, i.e., $|\beta| h<\mu$, and if $\{v, p\} \in\left[H^{2}(\Omega)\right]^{d} \times$ $H^{1}(\Omega)$, one easily shows that the choice $\delta=0$ and $\alpha \sim h^{2}$ leads to optimal a priori error estimates in the energy norm by Theorem 4.8. An error estimate in the $L^{2}$-norm for the velocities may then be recovered using a standard Nitsche duality argument.

\section{Variants of local projection stabilization.}

5.1. Local projection in streamlined direction. It should also be noted that from the practical viewpoint it may be more advantageous to use the streamlined derivative in the part of the subgrid model acting on the velocity in order to minimize crosswind diffusion; for instance,

$$
\begin{aligned}
S_{h}^{\beta}(u, \varphi):= & \left(\delta \bar{\varkappa}_{h}(\beta \cdot \nabla) v, \bar{\varkappa}_{h}(\beta \cdot \nabla) \psi\right)+\left(\delta \bar{\varkappa}_{h} \operatorname{div} v, \bar{\varkappa}_{h} \operatorname{div} \psi\right) \\
& +\left(\alpha \bar{\varkappa}_{h} \nabla p, \bar{\varkappa}_{h} \nabla \xi\right) .
\end{aligned}
$$

The following lemma states the fact that the proposed stabilization term (5.1) involving only diffusion in streamlined direction can be bounded by the triple norm. As a consequence, taking (5.1) does not affect the order of the numerical scheme.

Lemma 5.1. If $\beta \in\left[W^{1, \infty}(\Omega)\right]^{d}$, then it holds for all $v \in V_{h}$ that

$$
\left\|\delta^{1 / 2} \bar{\varkappa}_{h}(\beta \cdot \nabla) v\right\| \leq C_{\beta}\|\{v, 0\}\|,
$$

where $C_{\beta} \sim \delta^{1 / 2}\|\beta\|_{W^{1, \infty}(\Omega)} \sigma^{-1 / 2}+\|\beta\|_{\infty}$.

Proof. The proof follows by adding and subtracting $\bar{\pi}_{h} \beta$, where $\bar{\pi}_{h}$ denotes the projection on $D_{h}^{v}$ (here denoting the space of piecewise constants on the macropatches, regardless of the approximation). We apply the triangle inequality and the $H^{1}$ stability of $\bar{\varkappa}_{h}$ :

$$
\begin{aligned}
\left\|\delta^{1 / 2} \bar{\varkappa}_{h}(\beta \cdot \nabla) v\right\| & \leq\left\|\delta^{1 / 2} \bar{\varkappa}_{h}\left(\left(\beta-\bar{\pi}_{h} \beta\right) \cdot \nabla\right) v\right\|+\left\|\delta^{1 / 2} \bar{\varkappa}_{h}\left(\left(\bar{\pi}_{h} \beta\right) \nabla\right) v\right\| \\
& \lesssim\left\|\delta^{1 / 2}\left(\left(\beta-\bar{\pi}_{h} \beta\right) \cdot \nabla\right) v\right\|+\left\|\delta^{1 / 2} \bar{\varkappa}_{h}\left(\left(\bar{\pi}_{h} \beta\right) \nabla\right) v\right\| .
\end{aligned}
$$

The second term on the right-hand side is simply bounded by

$$
\begin{aligned}
\left\|\delta^{1 / 2} \bar{\varkappa}_{h}\left(\left(\bar{\pi}_{h} \beta\right) \nabla\right) v\right\| & \leq\|\beta\|_{\infty}\left\|\delta^{1 / 2} \bar{\varkappa}_{h} \nabla v\right\| \\
& \leq\|\beta\|_{\infty} S_{h}(u, u)^{1 / 2} .
\end{aligned}
$$


The first term can be estimated by the approximation property of $\bar{\pi}_{h}$ and a local inverse inequality:

$$
\begin{aligned}
\left\|\left(\left(\beta-\bar{\pi}_{h} \beta\right) \cdot \nabla\right) v\right\| & \lesssim \sum_{K \in \mathcal{T}_{2 h}}\|\bar{\varkappa} \beta\|_{K, \infty} h_{K}^{-1}\|v\|_{K} \\
& \leq\|\beta\|_{W^{1, \infty}(\Omega)}\|v\| .
\end{aligned}
$$

This gives

$$
\begin{aligned}
\left\|\delta^{1 / 2} \bar{\varkappa}_{h}(\beta \cdot \nabla) v\right\| & \lesssim\|\beta\|_{\infty} S_{h}(u, u)^{1 / 2}+\delta^{1 / 2}\|\beta\|_{W^{1, \infty}(\Omega)}\|v\| \\
& \leq C_{\beta}\|\{v, 0\}\| \quad \square
\end{aligned}
$$

Note that this result is valid immediately (without any assumptions on $\beta$ ) if the form (5.1) is used in the definition of the triple norm.

5.2. Projection onto a coarser mesh. As a further alternative we may use the nodal interpolant $\pi_{h}: Q_{h} \rightarrow Q_{2 h}$ and take as fluctuation filter $\varkappa_{h}:=i-\pi_{h}$. The stabilization term for the Oseen system can now be taken as

$$
S_{h}(u, \varphi):=\left(\delta \nabla \varkappa_{h} v, \nabla \varkappa_{h} \psi\right)+\left(\alpha \nabla \varkappa_{h} p, \nabla \varkappa_{h} \xi\right) .
$$

When the triple norm $\|\cdot\|$ is designed with the term $S_{h}(\cdot, \cdot)$, the coercivity property of Lemma 4.1 and the perturbed Galerkin orthogonality of Lemma 4.2 still hold for this variant. Also the estimate in Lemma 4.7 is still valid if we assume that $u \in H^{2}(\Omega)^{d+1}$ holds in order to be able to apply the nodal interpolant on $u$.

This variant can be considered as a generalization of the concept for advection equations of Guermond [11, 14].

5.3. Relation to classical stabilized methods. In this section, we will show the relation between the local projection method (LPS) analyzed in this paper and the GLS method or the residual-free bubble method. A key feature of the proposed method is the weak consistency: The fact that the stabilization enjoys the right asymptotic behavior without strong consistency allows us to decouple the stabilization of the pressure and the velocities and, even more importantly, allows us to decouple the stabilization from time-stepping terms and source terms. However, to show the relation to the GLS we will reintroduce the strong consistency. Our aim is to show that using the local projection stabilization we may in fact use GLS on the fine scales only, whereas the coarse scales are stable thanks to the interaction between coarse and fine scales. To this end we consider the full residual

$$
\rho(u):=\sigma v+\operatorname{div}(\beta \otimes v)-\mu \Delta v+\nabla p
$$

in the stabilization

$$
S_{g l s}\left(u_{h}, \varphi\right):=\left(\delta \bar{\varkappa}_{h} \rho\left(u_{h}\right), \bar{\varkappa}_{h} \rho(\varphi)\right)_{h}+\left(\delta \bar{\varkappa}_{h} \operatorname{div} v_{h}, \bar{\varkappa}_{h} \operatorname{div} \psi\right)
$$

where $(\cdot, \cdot)_{h}:=\sum_{K}(\cdot, \cdot)_{K}$. To make the formulation strongly consistent we perturb the right-hand side and obtain

$$
A\left(u_{h}, \varphi\right)+S_{g l s}\left(u_{h}, \varphi\right)=\left(f, \psi+\delta \bar{\varkappa}_{h} \rho(\varphi)\right)_{h} \quad \forall \varphi \in X_{h} .
$$

The consistency follows, and we note that for the exact solution $u$ we have

$$
S_{g l s}(u, \varphi)-\left(f, \delta \bar{\varkappa}_{h} \rho(\varphi)\right)_{h}=\left(\delta \bar{\varkappa}_{h}(\rho(u)-f), \bar{\varkappa}_{h} \rho(\varphi)\right)_{h}=0 .
$$


We have thus reformulated the local projection method as a GLS formulation with the stabilization acting only as a filter on the small scales. An important difference, however, is that the local projection approach using (5.4) does not impose any artificial boundary conditions on the solution contrary to the case of residual-free bubbles. This should be a definite advantage for nonlinear problems. We take as triple norm

$$
\|u\|_{g l s}:=\left(\left\|\sigma^{1 / 2} v\right\|^{2}+\left\|\mu^{1 / 2} \nabla v\right\|^{2}+\left\|\delta^{\frac{1}{2}} \bar{\varkappa}_{h}(\beta \cdot \nabla v+\nabla p)\right\|^{2}\right)^{1 / 2} .
$$

Note that we still have coercivity. In fact, after minor modifications, Theorem 4.8 remains true for (5.4).

LEMMA 5.2. It holds that

$$
\left\|u-u_{h}\right\|_{g l s} \lesssim h^{r+\frac{1}{2}}\left(\|v\|_{r+1}+\|p\|_{r+1}\right)
$$

if $\delta \lesssim \min \left(\frac{h}{\mu}, \frac{1}{\sigma}\right)$ with a constant depending on the constant in the $L^{2}$-stability of the projection $\overline{\bar{\varkappa}}_{h}$ and the constant in the inverse inequality.

Proof. We first note that

$$
\begin{aligned}
S_{g l s}\left(u-j_{h} u, u-j_{h} u\right)= & \left(\delta \bar{\varkappa}_{h} \rho\left(u-j_{h} u_{h}\right), \bar{\varkappa}_{h} \rho\left(u-j_{h} u\right)\right)_{h} \\
& +\left(\delta \bar{\varkappa}_{h} \operatorname{div}\left(v-j_{h} v_{h}\right), \bar{\varkappa}_{h} \operatorname{div}\left(v-j_{h} v\right)\right)
\end{aligned}
$$

has the right asymptotic, which is immediate, assuming optimal approximation for the second derivatives. One may then show, using the stability of the local projection and standard inverse inequalities, that provided $\delta$ satisfies the upper bound in the supposition, there holds

$$
\frac{1}{2}\left\|\delta^{\frac{1}{2}} \bar{\varkappa}_{h}\left(\beta \cdot \nabla v_{h}+\nabla p_{h}\right)\right\|^{2}-\frac{1}{2}\left\|\sigma^{1 / 2} v_{h}\right\|^{2}-\frac{1}{2}\left\|\mu \nabla v_{h}\right\|^{2} \lesssim S_{g l s}\left(u_{h}, u_{h}\right) .
$$

The proof now follows from (4.14) in the following fashion (considering here only the modified terms):

$$
\begin{aligned}
A(\eta, \xi) & \leq\|\eta\|_{g l s}\|\xi\|_{g l s}-\left(v-j_{h} v, \beta \cdot \nabla \xi^{v}+\nabla \xi^{p}\right) \\
& \leq\|\eta\|_{g l s}\|\xi\|_{g l s}-\left(\delta^{-1 / 2}\left(v-j_{h} v\right), \delta^{1 / 2} \bar{\varkappa}_{h}\left(\beta \cdot \nabla \xi^{v}+\nabla \xi^{p}\right)\right) \\
& \leq\left(\|\eta\|_{g l s}+\left\|\delta^{-1 / 2}\left(v-j_{h} v\right)\right\|\right)\|\xi\|_{g l s} .
\end{aligned}
$$

5.4. Extension to triangular meshes. Until now, we have considered meshes $\mathcal{T}_{h}$ with quadrilateral (or hexaedral) elements. The corresponding finite elements are $d$-linear $(r=1)$ or $d$-quadratic $(r=2)$. The question came up whether the described method is applicable also on elements with triangles $(d=2)$ or tetraedrons $(d=3)$. Of course, the definition of the method carries over to those triangulations without any modification if the patches are defined properly. It has to be assured that for a patch $K \in \mathcal{T}_{h}$, test functions with support inside $K$ do exist.

Bisection on triangles creates four smaller triangles out of one triangle; see Figure 5.1(a). Since no inner points are created, this strategy would not work in the case $r=1$ : The spaces $V_{h}(K)$ used in Lemma 4.3 would be empty in this particular case. However, possible patches are sketched in Figure 5.1(b) and (c). In the case $r=2$ and $d=2$, bisection leads to spaces $V_{h}(K)$ of dimension three corresponding to the three internal edges inside the patch; see Figure 5.1(a). The space $D_{h}(K)$ has the same dimension and can be represented by test functions corresponding to the three nodes of $K$. Hence, we have $\operatorname{dim} D_{h}(K) \leq \operatorname{dim} V_{h}(K)$, and the mapping $\pi_{K}$ can be defined injective. A similar situation occurs for $d=3$ and $r=2$ : $\operatorname{dim} V_{h}(K)=6$ and $\operatorname{dim} D_{h}(K)=4$. 


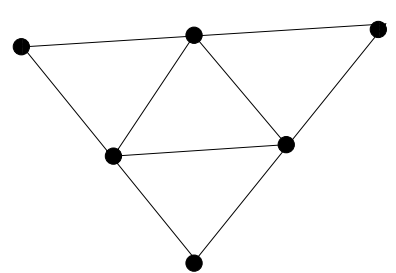

(a)

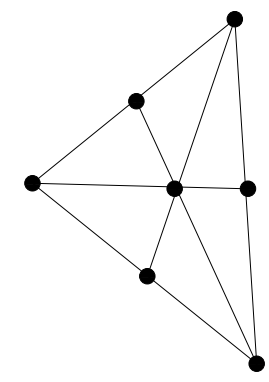

(b)

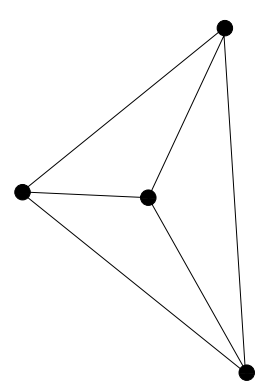

(c)

FIG. 5.1. Possible patches of triangles: (a) bisection does not create inner nodes and is therefore not suitable for LPS in the case $r=1$; (b) and (c) create inner nodes.

6. Link to the variational multiscale method. Today one of the major challenges in computational fluid dynamics is the accurate computation of different quantities in turbulent flow. Recently, several new approaches have been proposed such as the dynamic multilevel methodology (DML) of Dubois, Jauberteau, and Temam [10] or the VMS method of Hughes, Mazzei, and Jansen [15]. In the latter work, reference is made to residual-free bubble techniques (see Brezzi and Russo [4]) and subgrid viscosity as introduced by Guermond [14] to motivate an approach to large eddy simulation (LES), where the turbulence model acts only on the fine scales. In the following section we will show how the local projection method may be cast in the VMS framework, leading to a stabilized finite element method suitable for high Reynolds number flows.

6.1. Variational formulation of the Navier-Stokes equations. Let $I:=$ $[0, T]$ be the time interval. The velocities are sought in the Bochner space $\mathcal{V}^{v}:=$ $H^{1}(I, V)$, and the pressure in $\mathcal{V}^{p}:=L^{2}(I, Q)$. The product space will be denoted by $\mathcal{V}:=\mathcal{V}^{v} \times \mathcal{V}^{p}$. The test functions are in the space $\mathcal{W}:=L^{2}(I, X)$. The $L^{2}$-scalar product over the space-time slab $\Omega_{T}:=\Omega \times I$ will be denoted by $(\cdot, \cdot)_{\Omega_{T}}$, and its norm by $\|\cdot\|_{\Omega_{T}}$. Introducing now the state vector $u=\{v, p\} \in \mathcal{V}$, we may write the standard variational formulation of the Navier-Stokes equations (2.1): Find $u \in \mathcal{V}$ such that $v(\cdot, 0)=v_{0}$ and

$$
B(u, \varphi)=\langle f, \varphi\rangle \quad \forall \varphi \in \mathcal{W},
$$

where $B(u, \varphi)$ is defined for $\varphi=\{\psi, \xi\}$ by

$$
B(u, \varphi):=\left(\partial_{t} v, \psi\right)_{\Omega_{T}}-(v \otimes v, \nabla \psi)_{\Omega_{T}}+(\mu \nabla v, \nabla \psi)_{\Omega_{T}}-(p, \operatorname{div} \psi)_{\Omega_{T}}+(\operatorname{div} v, \xi)_{\Omega_{T}} .
$$

6.2. Separation of scales on the continuous level. In the VMS formulation as introduced in [15], a scale separation is performed and the turbulence model acts only on the finer scales. However, as always in turbulence modeling certain model assumptions on the interaction between the scales are made.

To clarify our model assumptions, we use the three-level partition proposed in Collis [9]. Hence we consider a scale separation in large resolved scales denoted by $\bar{v}$, small resolved scales denoted by $\tilde{v}$, and unresolved scales denoted by $\hat{v}$. The solution space is partitioned in a corresponding manner:

$$
\mathcal{V}=\overline{\mathcal{V}} \oplus \tilde{\mathcal{V}} \oplus \hat{\mathcal{V}}
$$


The function space $\mathcal{W}$ is partitioned similarly, $\mathcal{W}=\overline{\mathcal{W}} \oplus \tilde{\mathcal{W}} \oplus \hat{\mathcal{W}}$, with corresponding test functions, for instance, $\bar{\varphi}=\{\bar{\psi}, \bar{\xi}\} \in \overline{\mathcal{W}}$. We now write the exact equations of motions for each scale:

$$
\begin{array}{ll}
B(u, \bar{\varphi})=\langle f, \bar{\varphi}\rangle & \forall \bar{\varphi} \in \mathcal{\mathcal { W }}, \\
B(u, \tilde{\varphi})=\langle f, \tilde{\varphi}\rangle & \forall \tilde{\varphi} \in \hat{\mathcal{W}}, \\
B(u, \hat{\varphi})=\langle f, \hat{\varphi}\rangle & \forall \hat{\varphi} \in \hat{\mathcal{W}} .
\end{array}
$$

Introducing the linearized Navier-Stokes operator

$$
\begin{aligned}
B^{\prime}\left(u, u^{\prime}, \varphi\right):= & \left(\partial_{t} v^{\prime}, \hat{\psi}\right)_{\Omega_{T}}-\left(v^{\prime} \otimes v+v \otimes v^{\prime}, \nabla \psi\right)_{\Omega_{T}} \\
& -\left(p^{\prime}, \nabla \cdot \psi\right)_{\Omega_{T}}+\left(\mu \nabla v^{\prime}, \nabla \psi\right)_{\Omega_{T}}+\left(\nabla \cdot v^{\prime}, \xi\right)_{\Omega_{T}},
\end{aligned}
$$

the Reynolds stress projection

$$
R(v, \psi):=(v \otimes v, \nabla \psi)_{\Omega_{T}},
$$

and the cross-stress projection operator

$$
C(v, \hat{v}, \psi):=(v \otimes \hat{v}+\hat{v} \otimes v, \nabla \psi)_{\Omega_{T}},
$$

we may reformulate the exact equations for each scale in a fashion that makes evident the coupling between the scales. Following Collis [9], the exact solution $\bar{v} \in \overline{\mathcal{V}}$ for the resolved large scales fulfills for all $\bar{\varphi} \in \overline{\mathcal{W}}$ the equation

$$
\begin{aligned}
B(\bar{u}, \bar{\varphi})+B^{\prime}(\bar{u}, \tilde{u}, \bar{\varphi})-R(\tilde{v}, \bar{\psi})= & \langle f, \bar{\varphi}\rangle \\
& -B^{\prime}(\bar{u}, \hat{u}, \bar{\varphi})-R(\hat{v}, \bar{\psi})+C(\tilde{v}, \hat{v}, \bar{\psi}) .
\end{aligned}
$$

The first line in (6.5) includes the influence of the resolved scales on the large scales, whereas the second line includes the influence of the unresolved scales on the large scales. In the same fashion, the small resolved scales $\tilde{v} \in \tilde{\mathcal{V}}$ fulfill for all $\tilde{\varphi} \in \tilde{\mathcal{W}}$

$$
\begin{aligned}
B^{\prime}(\bar{u}, \tilde{u}, \tilde{\varphi})-R(\tilde{v}, \tilde{\psi})= & \langle f, \tilde{\varphi}\rangle-B(\bar{u}, \tilde{\varphi}) \\
& -B^{\prime}(\bar{u}, \hat{u}, \tilde{\varphi})-R(\hat{v}, \tilde{\psi})+C(\tilde{v}, \hat{v}, \tilde{\psi}) .
\end{aligned}
$$

The unresolved scales $\hat{v} \in \hat{\mathcal{V}}$ finally satisfy the following equation for all $\hat{\varphi} \in \hat{\mathcal{W}}$

$$
B^{\prime}(\bar{u}+\tilde{u}, \hat{u}, \hat{\varphi})+R(\hat{v}, \hat{\psi})=\langle f, \hat{\varphi}\rangle-B(\bar{u}+\tilde{u}, \hat{\varphi}) .
$$

It follows that the equation for the unresolved scales is driven by the residual of the resolved scales. With the equations written in this form it is easy to state the modeling assumptions as follows:

(M1) The unresolved scales $\hat{v}$ have no "direct" influence on the large scales. This means that the second line of (6.5) is set to zero:

$$
-B^{\prime}(\bar{u}, \hat{u}, \bar{\varphi})-R(\hat{v}, \bar{\psi})+C(\tilde{v}, \hat{v}, \bar{\psi})=0 \quad \forall \bar{\varphi} \in \overline{\mathcal{W}} .
$$

(M2) The influence of the unresolved scales on the small scales is modeled by an artificial viscosity term

$$
S: \mathcal{X} \times \mathcal{X} \rightarrow \mathbb{R}
$$

with $\mathcal{X}:=(\overline{\mathcal{V}} \oplus \tilde{\mathcal{V}}) \cup(\overline{\mathcal{W}} \oplus \tilde{\mathcal{W}})$, acting only on the small resolved scales. Hence we assume in (6.6) that for $\tilde{\varphi} \in \mathcal{\mathcal { W }}$

$$
S(\tilde{u}, \tilde{\varphi}) \approx B^{\prime}(\bar{u}, \hat{u}, \tilde{\varphi})+R(\hat{v}, \tilde{\psi})-C(\tilde{v}, \hat{v}, \tilde{\psi}) .
$$


The first modeling assumption (M1) can be expected to hold true when the main features of the flow are resolved. This is the large eddy assumption. The second modeling assumption (M2) implies that the unresolved scales only have the effect of dissipating energy from the small resolved scales. Heuristically one may argue that if assumption (M1) is satisfied, then the exact form or size of the subgrid model is of less importance as long as it allows for a sufficient rate of dissipation of energy from the resolved small scales to the unresolved scales. Insufficient dissipation may cause buildup of energy in high frequency modes (by the conservation properties of the Galerkin method) leading to spurious oscillations. Excessive dissipation will cause too much damping of the resolved small scales leading to poorer resolution of the large scales through the Reynolds stress coupling.

Using these modeling assumptions and the $L^{2}$-projection $\Pi v_{0}$ of the initial conditions onto the resolved scales $\overline{\mathcal{V}}^{v} \oplus \tilde{\mathcal{V}}^{v}$ we arrive at the formulation $(\bar{v}+\tilde{v})(\cdot, 0)=\Pi v_{0}$ and

$$
\begin{array}{lll}
B(\bar{u}+\tilde{u}, \bar{\varphi}) & =\langle f, \bar{\varphi}\rangle \quad \forall \bar{\varphi} \in \overline{\mathcal{W}} \\
B(\bar{u}+\tilde{u}, \tilde{\varphi})+S(\tilde{v}, \tilde{\varphi}) & =\langle f, \tilde{\varphi}\rangle \quad \forall \tilde{\varphi} \in \tilde{\mathcal{W}}
\end{array}
$$

We choose the subgrid viscosity term to be coercive on the small resolved scales $\tilde{u}$, $S(\tilde{u}, \tilde{u}) \geq c\|\nabla \tilde{u}\|^{2}$ for all $\tilde{u} \in \mathcal{W}$, symmetric $S(u, \varphi)=S(\varphi, u)$ for all $u, \varphi \in \mathcal{X}$, and such that it vanishes on the large resolved scales

$$
S(\cdot, \bar{\varphi})=0 \quad \forall \bar{\varphi} \in \overline{\mathcal{W}} \cup \overline{\mathcal{V}} .
$$

6.3. Separation of scales on the discrete level. We introduce some finite element approximation $\mathcal{V}_{h}$ of $\mathcal{V}$ that will represent the resolved scales $\mathcal{V}_{h}=\overline{\mathcal{V}} \oplus \tilde{\mathcal{V}}$. This space is then decomposed into large and small resolved scales by choosing $\overline{\mathcal{V}}=\mathcal{V}_{H}$, where $\mathcal{V}_{H} \subset \mathcal{V}_{h}$. To indicate its dependence on $h$, we equip the subgrid viscosity with a subscript, $S_{h}(\cdot, \cdot)$. The same discrete space is used for the test space $\mathcal{W}_{h}=\overline{\mathcal{W}} \oplus \tilde{\mathcal{W}}$. The discrete version of (6.9) becomes the following: Find $u_{h} \in \mathcal{V}_{h}$ such that $v_{h}(\cdot, 0)=$ $\pi v_{0}$ and

$$
B\left(u_{h}, \varphi\right)+S_{h}\left(\tilde{u}_{h}, \tilde{\varphi}\right)=\langle f, \varphi\rangle \quad \forall \varphi \in \mathcal{W}_{h}
$$

or, using the scale separation property $(6.10)$ of $S_{h}(\cdot, \cdot)$,

$$
B\left(v_{h}, \varphi\right)+S_{h}\left(u_{h}, \varphi\right)=\langle f, \varphi\rangle \quad \forall \varphi \in \mathcal{W}_{h} .
$$

Note also that by the properties of $S_{h}(\cdot, \cdot)$ we have Galerkin orthogonality for the discretization error $u-u_{h}$ on the large resolved scales:

$$
B\left(u-u_{h}, \bar{\varphi}\right)=0 \quad \forall \bar{\varphi} \in \mathcal{W}_{H} .
$$

Let us partition the time interval $I$ into subintervals $I_{n}=\left(t_{n-1}, t_{n}\right], n=1, \ldots, N$, with $0=t_{0}<t_{1}<\cdots<t_{N}=T$ and $\tau_{n}:=t_{n}-t_{n-1}$. We also introduce the space time slabs $Q_{n}:=I_{n} \times \Omega$. As the time integration scheme, we use the Crank-Nicholson scheme. It means that we choose piecewise $d$-linears for the ansatz functions and as test spaces piecewise constants (discontinuous), precisely,

$$
\mathcal{V}_{h}=P_{\tau}^{1}\left(I, X_{h}\right), \quad \mathcal{W}_{h}=P_{\tau}^{0}\left(I, X_{h}\right) .
$$


The spaces $\mathcal{V}_{H}$ and $\mathcal{W}_{H}$ are defined analogously by using $X_{H}$. With these finite element spaces we now propose the following finite element method: Find $u_{h} \in u_{0}+\mathcal{V}_{h}$, so that in the $n$th time step it holds for the restriction $u^{n}=\left\{v^{n}, p^{n}\right\}:=\left.u_{h}\right|_{I_{n}}$ :

$$
A_{n}\left(u^{n}, \varphi\right)+S_{h}\left(u^{n}, \varphi\right)=g_{n}\left(u^{n-1}, \varphi\right) \quad \forall \varphi \in \mathcal{W}_{h},
$$

with

$$
\begin{aligned}
A_{n}(u, \varphi) & :=\left(\tau_{n}^{-1} v, \psi\right)-(v \otimes v, \nabla \psi)-(p, \operatorname{div} \psi)+(\operatorname{div} v, \xi)+(\mu \nabla v, \nabla \psi), \\
g_{n}(u, \varphi) & :=\langle f, \varphi\rangle+\left(\tau_{n}^{-1} v, \psi\right)-(\mu \nabla v, \nabla \psi)+(v \otimes v, \nabla \psi)-S_{h}(u, \varphi) .
\end{aligned}
$$

As mentioned before, a widely used linearization of (6.14) is the Oseen linearization (2.3) with $\sigma:=\tau_{n}^{-1}$ and $\beta$ a suitable approximation on $v^{n}$ (for instance, the last iterate in the nonlinear iteration).

With these notations, we may take as subgrid model (3.2) or (5.3) with parameters $\delta$ and $\alpha$ depending on $h$. If triangular or tetraedral meshes are used, both subgrid operators satisfy (6.10) exactly and the analysis stated before shows that they are equivalent. On quadrilateral and hexaedral meshes, the stabilization (5.3) also satisfies (6.10) exactly. If version (3.2) is used on quadrilateral meshes, a small residual may remain due to the cross-term of $Q_{1}$ and $Q_{2}$ elements. Consequently, we do not have exact scale separation for (3.2) on quadrilaterals and hexahedrons.

7. Numerical example. Finally, we show numerical examples of this stabilization strategy. As the first step, the convergence rates of $v, \nabla v$ and of $p$ in $L^{2}$ are checked numerically on tensor grids and on locally refined meshes. In the next step, we investigate the difference of the kinetic energy of a nonstationary driven cavity flow with the numerical dissipation.

7.1. Convergence order for an exact Navier-Stokes solution. In order to check that the theoretical proven convergence order is also obtained numerically, we consider a stationary Navier-Stokes problem with known exact smooth solution in the unit square $\Omega:=(0,1)^{2}$ :

$$
\begin{aligned}
v_{x}(x, y) & :=-\cos (x) \sin (y)\left(2 \pi^{2}+1\right), \\
v_{y}(x, y) & :=\sin (x) \cos (y)\left(2 \pi^{2}-1\right), \\
p(x, y) & :=2(\cos (x)+\cos (y)) .
\end{aligned}
$$

The right-hand side $f$ is obtained by applying the Navier-Stokes operator to this solution. The solution is independent of the viscosity $\nu$ since the Laplacian applied to $v$ vanishes; $\Delta v=0$. However, this is not the case for the discrete solutions. The viscosity is set to $\nu=10^{-6}$ so that this is smaller than the mesh size.

We investigate the convergence order of $v$ in $L^{2}$ and in the $H^{1}$ seminorm, and for the pressure the $L^{2}$-error. Theorem 4.8 assures at least

$$
\left\|\nu \nabla\left(v-v_{h}\right)\right\| \leq\left\|u-u_{h}\right\|=\mathcal{O}\left(h^{r+1 / 2}\right) .
$$

Since $\nu<h$, we expect at least $\mathcal{O}\left(h^{r-1 / 2}\right)$ for $\left\|\nabla\left(v-v_{h}\right)\right\|$. Due to Theorem 4.9, we get the same order of convergence for $\left\|p-p_{h}\right\|$. With a standard duality argument we obtain one order more for the $L^{2}$-error in the velocities. This expectation is summed up in Table 7.1 (second column with label "theoretically") for the case $r=2$.

Let us first consider the case of equidistant tensor grids; see Figure 7.1 and the third column of Table 7.1. We clearly observe for this example the superconvergence behavior. 
TABLE 7.1

Theoretical and practical convergence rates for different quantities. The practical convergence rates summarize the results of the numerical example.

\begin{tabular}{c|ccc}
\hline & Theoretically & $\begin{array}{c}\text { Practice } \\
\text { Uniform meshes }\end{array}$ & $\begin{array}{c}\text { Practice } \\
\text { Nonuniform meshes }\end{array}$ \\
\hline \multirow{*}{*}{$* \nabla\left(v-v_{h}\right) \|$} & $h^{1.5}$ & $h^{2}$ & $h^{1.8}$ \\
$\left\|v-v_{h}\right\|$ & $h^{2.5}$ & $h^{3}$ & $h^{2.6}$ \\
$\left\|p-p_{h}\right\|$ & $h^{1.5}$ & $h^{2}$ & $h^{1.9}$ \\
\hline
\end{tabular}
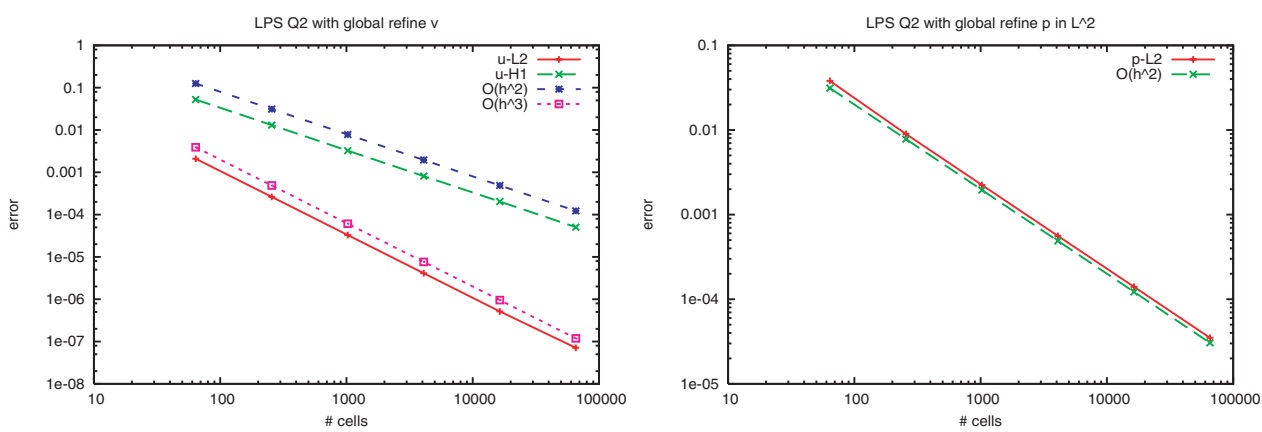

FIG. 7.1. Convergence for stabilized biquadratic elements $\left(Q_{2}\right)$ on equidistant tensor grids in dependence of the number of cells. Left: $\left\|v-v_{h}\right\|=\mathcal{O}\left(h^{3}\right)$ and $\left\|\nabla\left(v-v_{h}\right)\right\|=\mathcal{O}\left(h^{2}\right)$. Right: $\left\|p-p_{h}\right\|=\mathcal{O}\left(h^{2}\right)$.
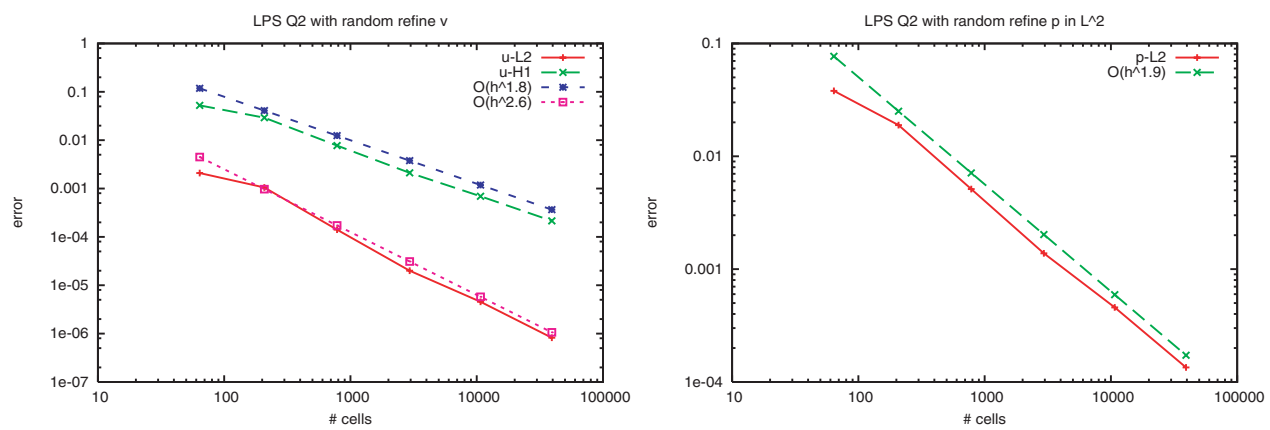

FIG. 7.2. Convergence for stabilized biquadratic elements $\left(Q_{2}\right)$ on randomly locally refined grids in dependence of the number of cells. Left: $\left\|v-v_{h}\right\|=\mathcal{O}\left(h^{2.6}\right)$ and $\left\|\nabla\left(v-v_{h}\right)\right\|=\mathcal{O}\left(h^{1.8}\right)$. Right: $\left\|p-p_{h}\right\|=\mathcal{O}\left(h^{1.9}\right)$.

On locally refined meshes superconvergence cannot be expected to such an extent. Therefore, we perform the same computations on a sequence of meshes which are obtained by refining approximately $50 \%$ of the cells by random. The meshes will be kept quasi-uniform with a ratio between the largest and the biggest mesh size bounded by 3 . In order to compare with tensor grids we consider the "global mesh size" $h:=n^{-1 / 2}$. In Figure 7.2 the convergence behavior is plotted on such locally refined meshes. The observed convergence rates are listed in the last column of Table 7.1. As expected, the convergence is reduced compared to tensor grids but is 


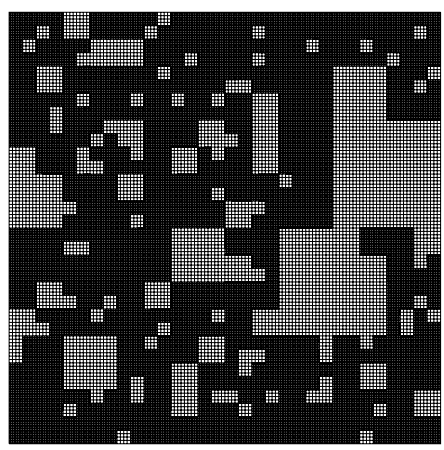

FIG. 7.3. One of the locally refined meshes used in the numerical example. The refinement is performed by random.

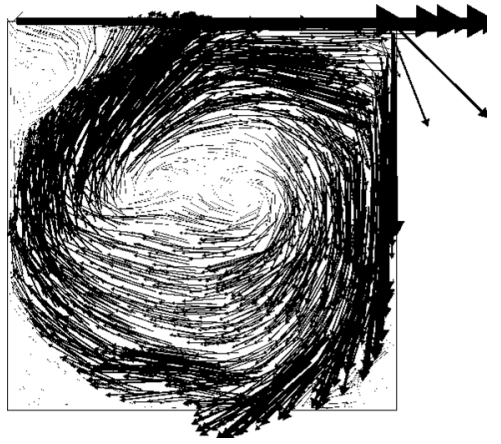

FiG. 7.4. Flow field of the driven cavity problem at $R e=10^{4}$ and $t=20 \mathrm{~s}$.

still better than the theoretical results. Note that this is not a fault of the proof but simply superconvergence on parts of the domain. We show one of the used meshes in Figure 7.3.

7.2. Driven cavity flow at $\boldsymbol{R e}=\mathbf{1 0}^{\mathbf{5}}$. The considered problem is a standard nonstationary driven cavity flow in the unit square $\Omega=(0,1)^{2} \subset \mathbb{R}^{2}$. As boundary conditions, we have for the vertical velocity component homogeneous Dirichlet conditions $v_{y}=0$ on $\partial \Omega$; for the horizontal velocity component we have $v_{x}=1$ on $\partial \Omega \cap\{y=1\}$ and $v_{x}=0$ elsewhere.

Although the configuration has been investigated for many years, it is still not clear when the transition to nonsteady flow exactly occurs. Auteri, Parolini, and Quartapelle [1] found the first Hopf bifurcation at about $R e=8018$ with a secondorder spectral projection method on a mesh with $160^{2}$ nodes. The computation of Peng, Shiau, and Hwang [17] shows at already Re $=7402 \pm 4$ the transition to a periodic solution. They state that the flow becomes "chaotic" for $R e>11000$. Further investigations can be found in $[7,13,20]$, each with their own critical Reynolds number for the first Hopf bifurcation, but all in the range between 7400 and 8375 . Hence, in order to guarantee a nonstationary flow we choose for our computations the Reynolds number $R e=10^{4}$.

The initial solution $\left.u\right|_{t=0}$ is chosen as the stationary solution at lower Reynolds number $\left(R e=10^{3}\right)$. The time step in the Crank-Nicholson scheme is chosen constant $\Delta t=0.05 \mathrm{~s}$. In Figure 7.4 the flow is shown at time instant $t=20 \mathrm{~s}$. 

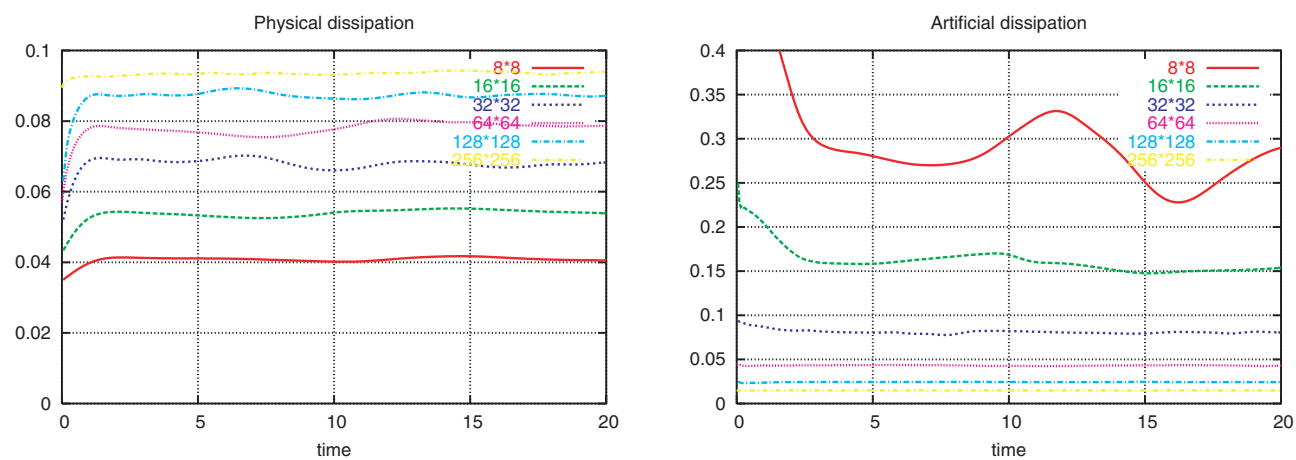

FIG. 7.5. Physical dissipation (left) and artificial dissipation (right) for the driven cavity problem at $\operatorname{Re}=10^{4}$.

TABLE 7.2

Mean values of physical and artificial dissipation and their ratio for the sequence of meshes.

\begin{tabular}{|l|ccccccc|}
\hline$\# Q_{2}$ cells & $8^{2}$ & $16^{2}$ & $32^{2}$ & $64^{2}$ & $128^{2}$ & $256^{2}$ & $512^{2}$ \\
\hline $\bar{e}_{\text {art }}^{h}$ & 0.299 & 0.162 & 0.0810 & 0.04278 & 0.0243 & 0.0149 & 0.00937 \\
$\bar{e}_{\text {phy }}^{h}$ & 0.0407 & 0.0536 & 0.0677 & 0.0782 & 0.0868 & 0.0934 & 0.0967 \\
Ratio & 7.34 & 3.02 & 1.20 & 0.55 & 0.28 & 0.16 & 0.097 \\
\hline
\end{tabular}
by

We compare the physical dissipation $e_{p h y}^{h}(t)$ and artificial dissipation $e_{\text {art }}^{h}(t)$, given

$$
\begin{aligned}
e_{p h y}^{h}(t) & :=\nu^{1 / 2}\left\|\nabla v_{h}(t)\right\|, \\
e_{a r t}^{h}(t) & :=S_{h}\left(u_{h}(t), u_{h}(t)\right)^{1 / 2}
\end{aligned}
$$

on a sequence of equidistant tensor grids with $8^{2}$ to $256^{2} Q_{2}$ cells (which corresponds to 3151875 dof's). In Figure 7.5, these two quantities are plotted in time. On the coarsest mesh considered, the physical dissipation stabilizes slightly above 0.04 . This quantity increases under mesh refinement, reaching nearly 0.1 on the mesh with $256^{2}$ $Q_{2}$ cells. At the same time, the artificial dissipation part $e_{a r t}^{h}$ decreases from about 0.3 to below of 0.01 .

For such time-dependent flows, physical meaningful quantities are time averages, denoted by overbars. For example, the mean of physical dissipation will be denoted by

$$
\bar{e}_{p h y}^{h}:=\frac{1}{T} \int_{0}^{T} e_{p h y}^{h}(t) d t .
$$

In Table 7.2, the averaged quantities and the ratio of artificial and physical dissipation are listed. While the artificial dissipation dominates the physical dissipation on the coarsest mesh, it becomes less than $10 \%$ on the finest mesh.

Although it cannot be expected that a solution $u_{H}$ on a coarse mesh $\mathcal{T}_{H}$ shows quantitatively the same information as the solution $u_{h}$ on the finest mesh $\mathcal{T}_{h}$, time averages should be comparable if the mesh is reasonably fine. We may hope that $\bar{u}_{H}$ coincides well with the $L^{2}$-projection of $\bar{u}_{h}$ onto $X_{H}$, denoted by $\Pi_{H} \bar{u}_{h}$. This is illustrated in Figures 7.6 and 7.7, where the isolines of the time-averaged velocity components are shown for the grid with $32^{2} Q_{2}$ cells. Although the mesh size differs by a factor of $2^{4}$, the two time averages coincide quite well. 

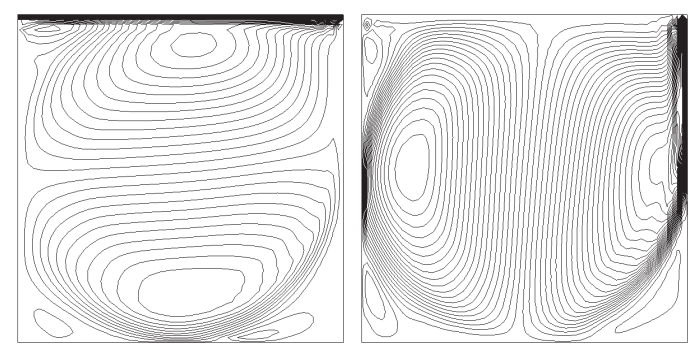

FIG. 7.6. Time-averaged velocities on a grid with $32 \times 32 Q_{2}$ cells; isolines of horizontal velocity component (left) and vertical velocity component (right).
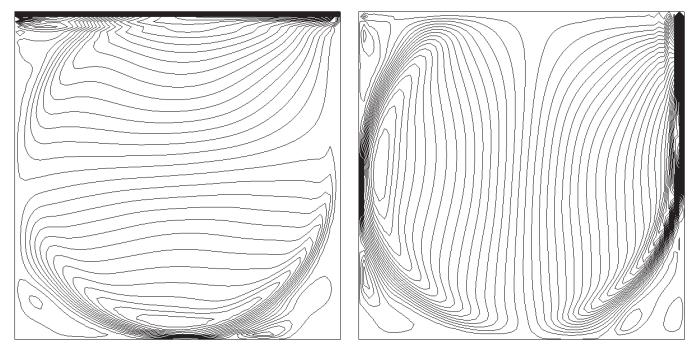

FIG. 7.7. Time-averaged solution on a grid with $512 \times 512 Q_{2}$ cells $L^{2}$-projected onto the finite element space with $32 \times 32 Q_{2}$ cells, $\Pi_{H} \bar{u}_{h}$; isolines of horizontal velocity component (left) and vertical velocity component (right).

8. Concluding remarks. We have proposed and analyzed a stabilized finite element method for the Oseen system based on local projections. To assure stability for the equal-order interpolation of velocity and pressure and for the case of high Reynolds number, a sufficient condition on the characteristic length scale of the subgrid model is established. This condition coincides with the condition for optimal-order convergence for the stabilized method when the underlying exact solution is smooth. We have discussed how the choices of stabilization parameters may influence the precision of the computation. Moreover, we have shown that the method can be formulated in a multiscale setting, hence rigorously establishing a link between stabilized methods and the VMS method for Navier-Stokes equations. We hope that this contribution will give additional insight into the close relationship between VMS and stabilized finite element methods. More extensive numerical simulations will be reported in a forthcoming paper.

\section{REFERENCES}

[1] F. Auteri, N. Parolini, And L. Quartapelle, Numerical investigation on the stability of singular driven cavity flow, J. Comput. Phys., 183 (2002), pp. 1-25.

[2] R. Becker And M. BraACK, A finite element pressure gradient stabilization for the Stokes equations based on local projections, Calcolo, 38 (2001), pp. 173-199.

[3] R. Becker AND M. BraAcK, A two-level stabilization scheme for the Navier-Stokes equations, in Numerical Mathematics and Advanced Applications, ENUMATH 2003, Springer-Verlag, Berlin, 2004, pp. 123-130.

[4] F. Brezzi And A. Russo, Choosing bubbles for advection-diffusion problems, Math. Models Methods Appl. Sci., 4 (1994), pp. 571-587.

[5] E. Burman and P. Hansbo, The edge stabilization method for finite elements in CFD, in Numerical Mathematics and Advanced Applications, M. Feistauer, ed., ENUMATH 2003, Springer-Verlag, Berlin, 2004. 
[6] E. Burman and P. Hansbo, A stabilized non-conforming finite element method for incompressible flow, Comp. Meth. Mech. Eng., submitted 2004.

[7] W. Cazemir, R. Verstappen, and A. Veldman, Proper orthogonal decomposition and lowdimensional models for the driven cavity flows, Phys. Fluids, 10 (1998), pp. 1685-1699.

[8] P. Clément, Approximation by finite element functions using local regularization, RAIRO, Anal. Numer., 9 (1975), pp. 77-84.

[9] S. Collis, Monitoring unresolved scales in multiscale turbulence modeling, Phys. Fluids, 13 (2001), pp. 1800-1806.

[10] T. Dubois, F. Jauberteau, and R. Temam, Incremental unknowns, multilevel methods and the numerical simulation of turbulence, Comput. Methods Appl. Mech. Engrg., 159 (1998), pp. 123-189.

[11] A. ERn And J.-L. Guermond, Theory and Practice of Finite Elements, Appl. Math. Sci. 159, Springer-Verlag, New York, 2004.

[12] V. Girault and P.-A. Raviart, Finite Elements for the Navier Stokes Equations, SpringerVerlag, Berlin, 1986.

[13] O. Goyon, High-Reynolds number solutions of Navier-Stokes equations using incremental unknowns, Comput. Methods Appl. Mech. Engrg., 130 (1996), pp. 319-335.

[14] J.-L. Guermond, Stabilization of Galerkin approximations of transport equations by subgrid modeling, M2AN Math. Model. Numer. Anal., 33 (1999), pp. 1293-1316.

[15] J. R. T. Hughes, L. Mazzei, and A. A. Oberai, The multiscale formulation of large eddy simulation: Decay of homogeneous isotropic turbulence, Phys. Fluids, 13 (2001), pp. 505511.

[16] J. M. Melenk and B. I. Wohlmuth, On residual-based a posteriori error estimation in hp-FEM, Adv. Comput. Math., 15 (2001), pp. 311-331.

[17] Y.-H. Peng, Y.-H. Shiau, And R. Hwang, Transition in a $2 d$ lid-driven cavity flow, Comput. \& Fluids, 32 (2003), pp. 337-352.

[18] T. C. Rebollo and A. D. Delgado, A unified analysis of mixed and stabilized finite element solutions of Navier-Stokes equations, Comput. Methods Appl. Mech. Engrg., 182 (2000), pp. 301-331.

[19] L. Scott And S. Zhang, Finite element interpolation of nonsmooth functions satisfying boundary conditions, Math. Comp., 54 (1990), pp. 483-493.

[20] G. Tiesinga, F. Wubs, And A. Veldman, Bifurcation analysis of incompressible flow in a cavity by the Newton-Picard method, J. Comput. Appl. Math., 140 (2002), pp. 751-772. 